\title{
The Development of Ultrafast Electron Microscopy
}

\author{
Sergei A. Aseyev ${ }^{1}$, Evgeny A. Ryabov ${ }^{1}$, Boris N. Mironov ${ }^{1}$ and Anatoly A. Ischenko ${ }^{2, *}$ \\ 1 Institute for Spectroscopy, Russian Academy of Sciences, ul. Fizicheskaya 5, Troitsk, Moscow 108840, Russia; \\ isanfemto@ya.ru (S.A.A.); ryabov@isan.troitsk.ru (E.A.R.); mironov@isan.troitsk.ru (B.N.M.) \\ 2 Department of Analytical Chemistry, RTU-MIREA-Russian Technological University, Institute of Fine \\ Chemical Technologies Named after M. V. Lomonosov, Vernadskogo avenue 86, Moscow 119571, Russia \\ * Correspondence: aischenko@yasenevo.ru
}

Received: 26 March 2020; Accepted: 7 May 2020; Published: 31 May 2020

\begin{abstract}
Time-resolved electron microscopy is based on the excitation of a sample by pulsed laser radiation and its probing by synchronized photoelectron bunches in the electron microscope column. With femtosecond lasers, if probing pulses with a small number of electrons-in the limit, single-electron wave packets-are used, the stroboscopic regime enables ultrahigh spatiotemporal resolution to be obtained, which is not restricted by the Coulomb repulsion of electrons. This review article presents the current state of the ultrafast electron microscopy (UEM) method for detecting the structural dynamics of matter in the time range from picoseconds to attoseconds. Moreover, in the imaging mode, the spatial resolution lies, at best, in the subnanometer range, which limits the range of observation of structural changes in the sample. The ultrafast electron diffraction (UED), which created the methodological basis for the development of UEM, has opened the possibility of creating molecular movies that show the behavior of the investigated quantum system in the space-time continuum with details of sub- $\AA$ spatial resolution. Therefore, this review on the development of UEM begins with a description of the main achievements of UED, which formed the basis for the creation and further development of the UEM method. A number of recent experiments are presented to illustrate the potential of the UEM method.
\end{abstract}

Keywords: ultrafast electron microscopy and diffraction; ultrafast structural dynamics; femtosecond pulsed lasers; ultrashort electron pulses; molecular movie

\section{Introduction}

The structural dynamics of matter induced by powerful pulsed laser radiation manifests itself in an atomic-molecular movie, the study of which is of great interest to modern science. To this end, it is necessary to ensure a high spatiotemporal resolution, which implies the development of special research methods. Direct observation of laser-stimulated structural changes of in any substance can only be implemented using quite short electron bunches or X-ray flashes, strictly synchronized with powerful light pulses irradiating this material [1-5]. The use of electron beams for probing thin films or gaseous media is preferable, and, in a number of cases, is the only possible tool, because the interaction cross section of fast electrons with matter is five to four orders magnitude greater compared to X-rays [2].

A pioneering experiment that was performed by a group of researchers in Moscow in the 1980s using a gas phase diffractometer [6] made it possible to combine the temporal resolution of a pulsed laser with a subatomic spatial resolution provided by probing the sample with an electron beam. The appearance on the market of powerful and reliable femtosecond lasers and the development of bright pulsed photoelectron sources based on them have determined a breakthrough in ultrafast electron diffraction and microscopy [1-5]. Prof. Miller with colleagues performed the first successful experiments using multielectron pulsed beams in which a subpicosecond time resolution in UED was 
achieved [7], and developed the fundamentals of femtosecond electron optics [8]. As a result, the use of UED made it possible to observe in real time the motion of atoms in matter during laser-induced chemical transformations, as well as to find experimentally the correlation between a number of structural functions, which underlies such disciplines as biology and chemistry [1-5].

At the beginning of the 21st century, scientists at the California Institute of Technology reported about the first series of experiments that were conducted on a transmission electron microscope modified to directly observe the structural dynamics of matter on a picosecond time scale, which was then extended to the femtosecond range, which marked the birth of ultrafast electron microscopy (UEM). If we appeal to the history of the development of classical electron microscopy, we can easily trace the tendency toward improving the resolving power of corresponding instruments [9]. Thus, whereas in the middle of the 20th century, the resolution of first electron microscopes was comparable with the resolution of their best optical analogs at that time, then, around the turn of the millennium, electron optics with careful aberration correction proved to be capable of providing the spatial resolution in this field even at a level of $\sim 0.8 \AA[9,10]$. It should be noted that the introduction of modern pulsed laser technology gave a powerful impetus to the development of electron microscopy [1-5].

By now, numerous works have been published that describe the development of UED and UEM methods. Thus, a review article on the possibility of creating atomic-molecular movies [5] traces the history of development of the method of ultrafast electron diffraction actually until 2017. Another review [11] presents the current state of time-resolved electron microscopy. The goal of this paper is, first, to illustrate the later development of UED, since 2018. To demonstrate the potential of this method, a number of recent investigations will be presented. Second, we will try to highlight here the capabilities of UEM, which, for whatever reason, were not covered, or were only briefly outlined in [11]. In contrast to a fairly recent review [11], we confine ourselves to the stroboscopic mode of operation of the time-resolved electron microscope, when it becomes possible to experimentally detect fast processes on pico-femto-attosecond scales.

\section{Illustrative Examples of UED Experimental Activity in 2018-2020}

In recent years, a great number of important observations of structural dynamics in matter have been made by the UED method using ultrashort probing electron pulses. Two main regimes of operation include stroboscopic and single-shot modes. In the stroboscopic fashion, repeatable ultrafast processes are probed by a train of photoelectron pulses, while in the single-shot mode irreversible laser-induced phenomena are studied by using individual multi-electron probing bunches. The conventional stroboscopic UED approach uses hundreds of ultra-short electron pulses to sample each time point of the delay in detecting the entire dynamics.

Here, it is worth dwelling on the problem of the brightness of a pulsed electron source. Atomic-molecular structures can be detected either (i) in reciprocal space using the electron diffraction method, or (ii) in real space in the imaging regime. In the first case, the signal increases as $N^{2}$, while, in the second case, it is proportional to $N$, where $N$ is determined by the number of unit cells in the probed volume of a crystalline sample [5]. In fact, electron diffraction is a much more sensitive method than real-time imaging [12], which, as applied to developed pulsed electron sources, makes it virtually the only possible tool to detect the atomic-molecular structure per one probe pulse. In this respect, we note that, for simple unit cells, containing $\sim 10$ atoms, at least $10^{4}$ electrons should be detected to obtain a diffraction pattern with an acceptable dynamic range. The required number of electrons increases approximately linearly with an increase in the number of atoms in the unit cell, which follows from their stationary electron diffraction data. Thus, for molecular systems consisting of $\sim 100$ atoms, $\sim 10^{5}$ electrons are already required, and about $10^{7}$ electrons to visualize proteins.

In practice, the number of electrons determines the signal-to-noise ratio in UED experiments. The milestone UED results on Al melting were obtained using 150 shots of 6000 electrons each, for a total of $\sim 10^{6}$ electrons per diffraction pattern [5]. This can be considered a good estimate for the minimum charge per bunch required to obtain a high-contrast electron diffraction pattern. 
The particular value of UED is also that this method makes it possible to ensure a femtosecond time resolution even in cases when each probe pulse contains up to $\sim 10^{6}-10^{7}$ electrons [5]. This can be achieved by using a cavity with a radiofrequency (RF) field [13], a magnetic sector, or a reflectron for compression of many electron bunches $[14,15]$. Moreover, an extremely compact scheme can be used for these purposes when the distance from the photocathode to the sample is minimized [16,17]. An alternative option is the passage to relativistic velocities, when a dense electron bunch does not actually have time to expand during its propagation to the substance under study $[17,18]$. Finally, we should mention the method of forming homogeneous electron pulses having the shape of a regular ellipsoid, for which the degree of compression can reach maximum possible value [19]. Under these conditions, the signal integration time is drastically reduced [5], and it even becomes possible to detect irreversible laser-induced processes. The use of the above approaches to create ultrashort dense electron pulses in a column of a modified transmission electron microscope faces serious difficulties caused by the industrial design of the device, which was originally designed to solve stationary problems.

The ultrafast electron diffraction (UED), which created the methodological basis for the development of UEM, has opened the possibility of creating molecular movies that show the behavior of the investigated quantum system in the space-time continuum with details of sub- $\AA$ spatial resolution. Therefore, this review on the development of UEM begins with a description of the main achievements of UED, which formed the basis for the creation and further development of the UEM method. In this section, we will illustrate the success of the development of pico- and femtosecond electron optics as applied to UED using recent experiments as an example.

The attraction of graphene is due to its unique properties, which, upon industrial production based on graphene oxide (GO), depend on the corresponding reduction processes. In fact, the understanding of these mechanisms is crucial for controlling the properties of a whole range of functional 2D systems. In the experiment in [20], the structure of a reduced compound that was obtained both upon heating of GO and upon its photoexcitation was recorded. In that work, the reduction mechanisms were determined based on UED data, ultrafast IR vibrational spectroscopy (laser pulse duration, $120 \mathrm{fs}$; tuning spectral range, $1000-4000 \mathrm{~cm}^{-1}$ ), and calculations using the density functional theory. Within the UED, the sample under study was pumped by femtosecond laser radiation at a wavelength of $266 \mathrm{~nm}$ and a repetition rate of $1 \mathrm{kHz}$ and was probed by a $\sim 1$-ps $75 \mathrm{keV}$ pulsed electron beam. The energy density in laser pulses exciting the sample reached $5 \mathrm{~mJ} / \mathrm{cm}^{2}$. It was found that, upon photoexcitation, oxygen atoms of epoxy groups were selectively removed from the GO basal plane, as distinct from the thermal reduction of hydroxyl and epoxy groups in the thermal regime, which, in turn, was determined by the selective character of the laser-induced excitation process of epoxy bonds. Work [20] may considerably contribute to the development of an effective GO reduction method and, thereby, extend the scope of applications of 2D systems.

The molecular photoisomerization process finds numerous applications for monitoring the structure of soft matter in various natural and artificial systems. However, the structural dynamics of molecules during isomerization is difficult to observe due to the rather complex character of this high-speed process. To solve this problem, the UED method was used in [21]. To probe a sample with a thickness of $\sim 60 \mathrm{~nm}$ deposited on a 30-nm SiN membrane, picosecond bunches containing $\sim 2 \times 10^{4}$ electrons with a kinetic energy of $75 \mathrm{keV}$ were used. The sample was pumped by femtosecond laser radiation at a wavelength of $266 \mathrm{~nm}$ with a repetition rate of up to $333 \mathrm{~Hz}$. The energy density in the laser pulses exciting the sample reached $0.5 \mathrm{~mJ} / \mathrm{cm}^{2}$. In this experiment, the ultrafast formation of liquid crystalline azobenzene molecules with a higher orientation took place under the action of linearly polarized pulsed UV laser radiation. It was found that this process was caused by trans-cis isomerization of examined molecules. As it turned out, these experimental data are consistent with molecular dynamics calculations, from which it follows that, after photoinduced isomerization, molecular structures are aligned in a strong linearly polarized light field. The data obtained are of undoubted interest for the development of photochemistry of soft matter. 
The UED-based approach enabled direct detection of ultrafast structural dynamics of molecules in the gas phase. However, in the case of a nonrelativistic electron beam, it turned out to be rather problematic to surmount the picosecond barrier, which limited the possibility of creating a molecular movie about a chemical reaction. To solve this problem, a UED experimental apparatus using relativistic electrons was created at the SLAC National Accelerator Laboratory [22]. The unique temporal resolution of this experimental scheme, equal to $65 \mathrm{fs}$, along with a spatial resolution of $0.63 \AA$ and a resolution in reciprocal space of $0.22 \AA^{-1}$, made it possible to visualize the main photochemical processes, such as the breaking of a chemical bond, ring opening, as well as the motion of a nuclear wave packet through a conical intersection $[23,24]$.

The molecular response to ultraviolet radiation is of interest not only from the fundamental point of view, but also because it most directly related both to the process of photosynthesis and to numerous applications. As is well known, all optical methods fail to directly detect the molecular structure, and alternative approaches are required. In the experiment in [23], using $\mathrm{CF}_{3} \mathrm{I}$ as an example, direct observation of the molecular structure was realized for the first time upon passage through a conical intersection of potential energy surfaces, for which a complex approach was used based on the combination of the relativistic UED method in the gas phase along with theoretical calculations. Irradiation of the molecular structure by femtosecond laser UV radiation initiated the dissociation of the C-I bond in an excited electronic state. The conical intersections of potential energy surfaces in which the excited electronic state and some other electronic state become degenerate are a sort of "funnels" that make it possible to transfer the quantum system from a state with electron excitation to an underlying excited electronic state or back to the ground state. In the recorded molecular movie on laser-induced dissociation of $\mathrm{CF}_{3} \mathrm{I}$ molecules [23], movements of the $\mathrm{CF}_{3}$ fragment clearly manifested themselves, which were accompanied by the elongation of $\mathrm{C}-\mathrm{F}$ bonds, with this stretching process being delayed in time with respect to the initial opening of the F-C-F bond. It was found that the atoms were rearranged within $100 \mathrm{fs}$ after reaching the conical intersection, where bifurcation of populations of electronic states took place. Upon passage through the conical intersection of the potential energy surfaces, a certain part of the population turned out to be transferred to underlying electronically excited states, which was accompanied by the restoration of the initial structure on a subpicosecond time scale. For a higher lying excited electronic state, the process of stretching of the $\mathrm{C}-\mathrm{I}$ bond with a characteristic time of $200 \mathrm{fs}$ was detected. In fact, each time as the nuclear wave packet returned to the conical intersection of the potential energy surfaces, a large portion of the population was transferred to underlying electronic states, which, in turn, led to quantum mechanical interference. The data obtained demonstrated the potential of the UED method for creating molecular movies.

In an experiment that was recently performed on a compact femtosecond electron diffractometer with an electron energy of $20 \mathrm{keV}$ at the Institute for Spectroscopy in Troitsk (Russia), the coherent dynamics of lattice vibrations in a $20 \mathrm{~nm}$ thick polycrystalline bismuth film was recorded. The pulsed heating of the Bi lattice due to irradiation of the sample by femtosecond laser radiation at a wavelength of $800 \mathrm{~nm}$ with a pulse energy density of $\sim 2 \mathrm{~mJ} / \mathrm{cm}^{2}$ was accompanied by the formation of high-frequency optical phonons, which were detected by the UED method. Fourier analysis of the data showed that the revealed modulation of the signal is created by the ensemble of modes corresponding to optical phonons with frequencies of 3,6 , and $9 \mathrm{THz}$. It was concluded that these peaks refer to the $A_{1 g}$ mode of bismuth, as well as to its first and second overtones. Due to the absence of published data on the generation of the third harmonic of the $A_{1 g}$ mode in this material, it was suggested that the obtained results pointed to the manifestation of quantum confinement in a thin film of bismuth, for which the Fermi wavelength reached $\sim 40 \mathrm{~nm}$ [25].

Previously [25], bulk and thin-film Bi samples have been studied comprehensively using femtosecond laser spectroscopy in reflection mode; however, the closeness of the characteristic thickness of a nanostructure to the penetration depth of the $800 \mathrm{~nm}$ radiation made it difficult to observe quantum confinement because of reflection of the probe laser beam from the back surface of the film. In principle, the UED method is capable of removing this limitation, which opens up the possibility of 
a detailed study of the quantum size effect $[26,27]$ in thin Bi films in a wide range of thicknesses using relativistic electron pulses, when the characteristic electron mean free path is $\sim 100 \mathrm{~nm}$.

In [28], the UED method (kinetic energy of electrons, $30 \mathrm{keV}$; duration of electron pulses, a few picoseconds) was used to study coherent acoustic phonons in a 30-nm sample of a Bi polycrystal. The results were analyzed using the model of a damped harmonic oscillator, in which the explosive force created by heated electrons of the solid substance triggered lattice oscillations. Based on the experimental data for the bismuth crystal, the electron-phonon interaction rate and the Grüneisen parameter corresponding to the electronic subsystem were determined [28].

In experiments of $[29,30]$, the laser-induced response from the lattice on the surface of a bismuth single crystal was detected by the UED method in reflection geometry. In [28], the (111) Bi surface was irradiated by femtosecond laser pulses with energy densities of up to $2 \mathrm{~mJ} / \mathrm{cm}^{2}$. The observed diffraction patterns corresponded to a significant change in the electron momentum in the range of $9.3 \AA^{-1} \leq \Delta k \leq 21.8 \AA^{-1}$. In the experiment, the diffraction signals, depending on $\Delta k$, decayed with characteristic times in the range of 5 to 12 ps, which was fairly well described by an exponential function in the Debye-Waller factor. Therefore, upon irradiation of the crystal with femtosecond laser pulses with moderate energy densities, the authors of [29] did not reveal any deviations from the isotropic Debye-Waller effect, which made it possible to use this model to analyze the thermal motion of atoms under these conditions. As follows from the data obtained in [30], the laser-induced process of heating the crystal volume proceeded much faster, in as little as a few picoseconds, compared with the excitation characteristic time of the vibrational motion of surface Bi atoms, equal to $12 \mathrm{ps}$. This demonstrated a weak coupling between 2D and 3D phonon modes, which, in turn, impeded the energy exchange between the surface and the bulk of the crystalline sample.

Spin transitions belong to basic molecular photophysical processes. A change in the spin states via the spin-orbit interaction affects the spatial distribution of the electron density of a solid substance, which is accompanied by a structural rearrangement of the molecular complex. In the experiment in [31], a $\left[\mathrm{Fe}^{\mathrm{II}}(\mathrm{bpy})_{3}\right]\left(\mathrm{PF}_{6}\right)_{2}$ single crystal, a prototype for the observation of the dynamics of spin crossover, was studied by the UED method in the single-photon excitation regime. For this purpose, a single-crystal sample with a thickness of $150 \mathrm{~nm}$ with an (2 10$)$ orientation was excited by 50-fs laser pulses at a wavelength of $400 \mathrm{~nm}$, an energy density per pulse of $\sim 5 \mathrm{~mJ} / \mathrm{cm}^{2}$, and a repetition frequency of $50 \mathrm{~Hz}$. The laser-induced dynamics in the solid was probed by $95 \mathrm{keV}, 270 \mathrm{fs}$ pulses containing approximately $7 \times 10^{4}$ electrons, which was achieved by compression of electron bunches in a radiofrequency field. As a result, a fast process with a characteristic time of about $450 \mathrm{fs}$ and a slower one with a characteristic time of $\sim 2.4$ ps were detected. Based on an analysis of the experimental data, key structural modes (ultrafast stretching of the $\mathrm{Fe}-\mathrm{N}$ bond accompanied by ligand displacements) were ascertained, which determined the trajectory of the laser-induced molecular reaction.

Interactions between the lattice and charge carriers in a solid can lead to phase transitions and ordering processes, which, in particular, are responsible for superconductivity, insulator-to-metal transition, and generation of charge density waves. These interactions also determine the electrical properties of the material and its thermal conductivity. Ultrafast Diffuse Electron Scattering (UEDS) has become a powerful laboratory tool for tracking the energy flux in the lattice through the entire Brillouin zone, as well as for deconvolution of interactions on a time scale. In [32], a methodology for interpreting UEDS signals to determine the populations of phonon modes in the Brillouin zone was presented. In a demonstration experiment, a $70 \mathrm{~nm}$ thick single-crystal graphite sample with the (2 10 ) orientation was excited by 35-fs laser pulses at a wavelength of $800 \mathrm{~nm}$, a pulse energy density of $\sim 12 \mathrm{~mJ} / \mathrm{cm}^{2}$, and a repetition rate of $1 \mathrm{kHz}$. The laser-induced dynamics in the solid substance was probed with $90 \mathrm{keV}, 150$ fs pulses containing approximately $10^{7}$ electrons, which was ensured by compression of electron bunches in the radio-frequency field. In that work, a resolution of $0.06 \AA^{-1}$ in the reciprocal space was achieved. From the analysis of experimental data, it was possible to determine the electron-phonon and phonon-phonon coupling constants for graphite. 
The author sof [33] described the study of vanadium dioxide by the UED method (pulsed electron beam parameters are close to those in [32]) in combination with time-resolved terahertz spectroscopy, which demonstrated an important example of controlling the properties of strongly correlated materials by light. The goal of such a complex experiment, combining two powerful research methods at the same time, is to ascertain a relationship between ultrafast laser-induced changes in optoelectronic properties and the lattice structure in $\mathrm{VO}_{2}$. In that work, the structure of a metastable monoclinic metal phase of a solid substance with antiferroelectric properties arising because of a thermally activated orbital-selective phase transition in the electronic subsystem was detected. Moreover, the relative contribution of the low-temperature monoclinic and high-temperature rutile phases to the response of the $50 \mathrm{~nm}$ vanadium dioxide film excited by powerful electromagnetic pulses was determined.

It is well known that, in physics of condensed matter, the coupling between charge carriers and phonons is responsible for a number of related processes. In the experiment in [34], the method of ultrafast diffuse electron scattering (the experimental scheme is close to that of [32]) was used to study such a coupling in the momentum-and-time continuum. In that work, a laser-induced response of a 1T-TiSe $e_{2}$ semimetal sample to selective doping by charge carriers was observed. The results indicate a strong exciton-phonon coupling in this dichalcogenide, and, also, unravel the main mechanism by which the transition to charge density waves is controlled.

\section{Imaging Ultrafast Coherent Phenomena with UEM}

The use of a transmission electron microscope adapted for time-resolved measurements (The article does not consider the "laser-free" approach, which, as was shown in [35], is currently able to provide the formation of 10 ps electron pulses in the column of a transmission electron microscope.) opens up the possibility of detecting irreversible laser-induced processes that proceed on a nanosecond time scale, when their imaging is implemented by single bunches containing up to $\sim 10^{8}$ electrons, and strictly reversible coherent phenomena in the signal integration regime due to integration of a large number of electronic pulses. In the stroboscopic regime, UEM provides ultrahigh spatiotemporal resolution by suppressing the influence of Coulomb repulsion [1,4].

Here, it will be appropriate to note the important contributions to the development of the stroboscopic technique for recording dynamic effects in solids that were made in the 20th century in the USSR [36] and in Germany [37]. The essence of this approach is as follows; a sample whose properties periodically vary in time at a fixed frequency of changes, should be irradiated by short electronic pulses at the same frequency and at a fixed phase. Then, the obtained image will "freeze" and will reflect the state of the substance under study at the moments of arrival of electronic pulses.

The first transmission electron microscope that was adapted for imaging ultrafast, laser-induced processes was created at the California Institute of Technology (United States) [1]. To illustrate its work, Figure 1 shows a diagram of such an instrument, which contains three main units:

(a) Unit of formation of a pulsed photoelectron beam of subpicosecond duration.

(b) Sample excitation unit by femtosecond laser radiation with subsequent probing of the spatiotemporal structure by ultrashort electron pulses.

(c) Position-sensitive 2D detection of electrons.

The modern UEM arrangement enables detecting ultrafast coherent processes in matter in real space in the imaging mode, by using the method of electron diffraction in reciprocal space, as well as by performing precise measurements of the kinetic energy of electrons after their interaction with the sample in the electron energy-loss spectroscopy (EELS) regime. The real-space mapping seems to be very valuable to study the structural dynamics of matter. In this case, it becomes possible to visualize and identify spatial inhomogeneities of the examined material, as well as to determine their local influence on fast processes, which is significantly more informative method, compared to diffraction, of study of laser-induced phenomena in materials containing defects, phase boundaries, and dislocations [39-41]. 


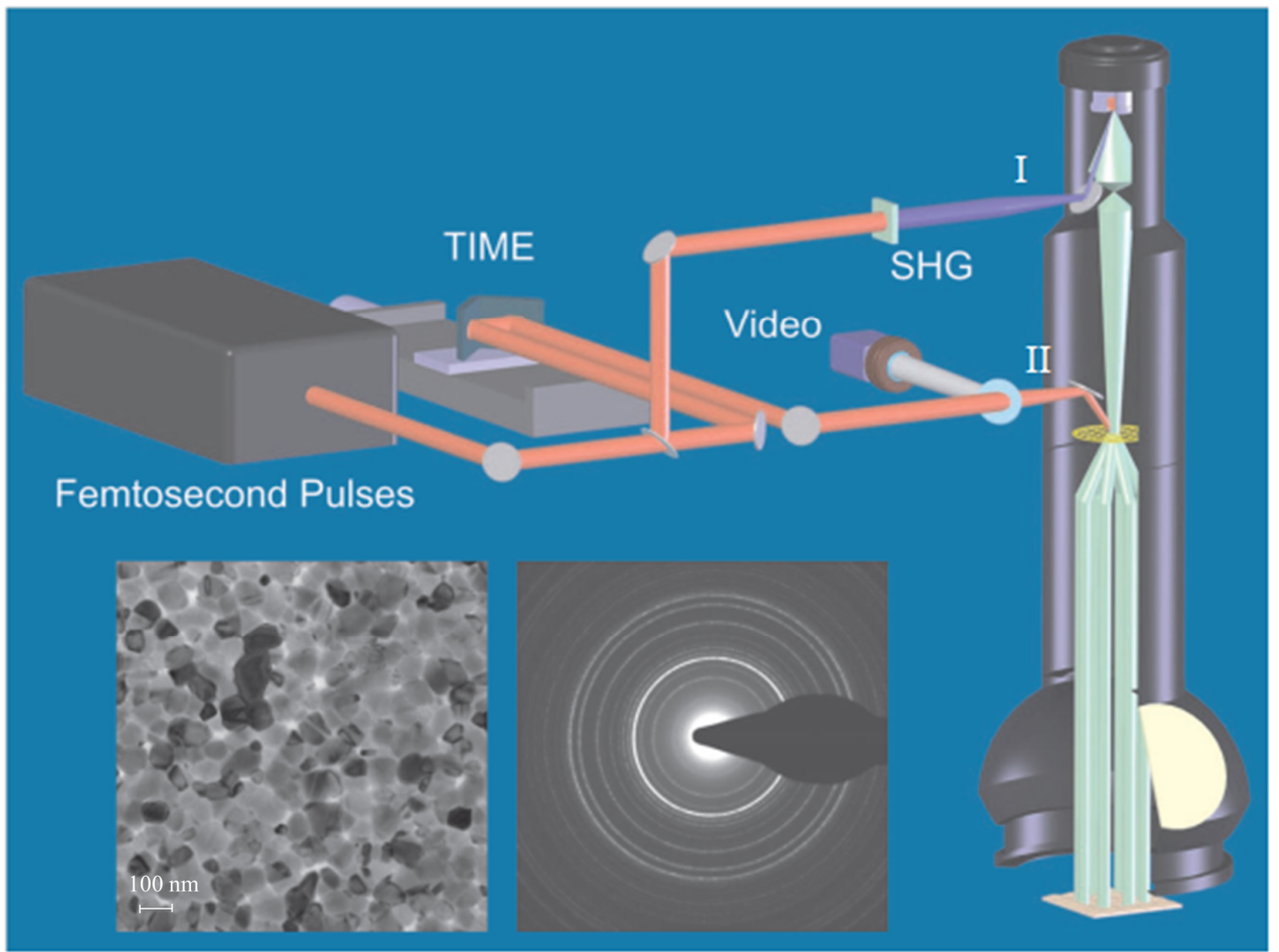

Figure 1. (Color online) Schematic of an ultrafast transmission electron microscope [38]. A commercially available transmission electron microscope that was originally designed for continuous operation with a thermionic cathode can be taken as a basis for creating such a setup. In order to adapt the industrial apparatus to UED requirements, it is necessary to ensure pulsed laser excitation of the sample (marked with symbol II), as well as the delivery of the laser radiation to the photocathode (marked with symbol I) to generate an ultrashort pulsed photoelectron beam. (Adapted from work in [38] with minor changes. Copyright (2006) National Academy of Sciences.)

Here, we will consider the stroboscopic imaging regime of repetitive laser-induced processes which, at a small number of electrons in the probe pulse, can provide ultrahigh temporal resolution. To emphasize the capabilities of this approach, we will present a number of illustrative examples.

\subsection{UEM Imaging of Laser-Induced Phase Transition}

Vanadium dioxide $\left(\mathrm{VO}_{2}\right)$ experiences a phase transition from a low-temperature monoclinic phase (M) to a high-temperature tetragonal rutile phase (R) at a temperature in the range of $\sim 67^{\circ} \mathrm{C}$. Since the time of its discovery, which was made almost half a century ago, this metal-insulator transition process has been the subject of numerous studies. In [38], the UEM method was used for this purpose for the first time. The experiment was performed on a G2 12 TWIN Tecnai transmission electron microscope with a kinetic energy of the electron beam of up to $120 \mathrm{keV}$, which was modified for time-resolved measurements (Figure 1). Ultrafast processes in a solid-state sample were probed using single-electron pulses with a repetition rate of $80 \mathrm{MHz}$ that were generated upon irradiation of the cathode by the second harmonic of a Ti:Sa laser at a wavelength of $338 \mathrm{~nm}$, while the sample was excited by the fundamental harmonic at a wavelength of $778 \mathrm{~nm}$ and a laser pulse duration of $120 \mathrm{fs}$. Precise synchronization of optical and photoelectron pulses was provided by a single master femtosecond laser for excitation and probing channels.

In a regime in which each probe pulse contains about one electron, there is practically no Coulomb repulsion, which ensures high spatial and $\sim 100 \mathrm{fs}$ time resolution. The sequence of snapshots obtained 
as a result of integration of the signal over very many pulses represents a movie about a laser-induced phase transition in $\mathrm{VO}_{2}$. In this experiment, the metal-insulator structural transition was clearly pronounced both in diffraction patterns and in the image regime (Figure 2), with the time of this phase transformation being roughly 3 ps, which, according to optical measurements, corresponded to a $200 \mathrm{~nm}$ thickness of the vanadium dioxide sample.
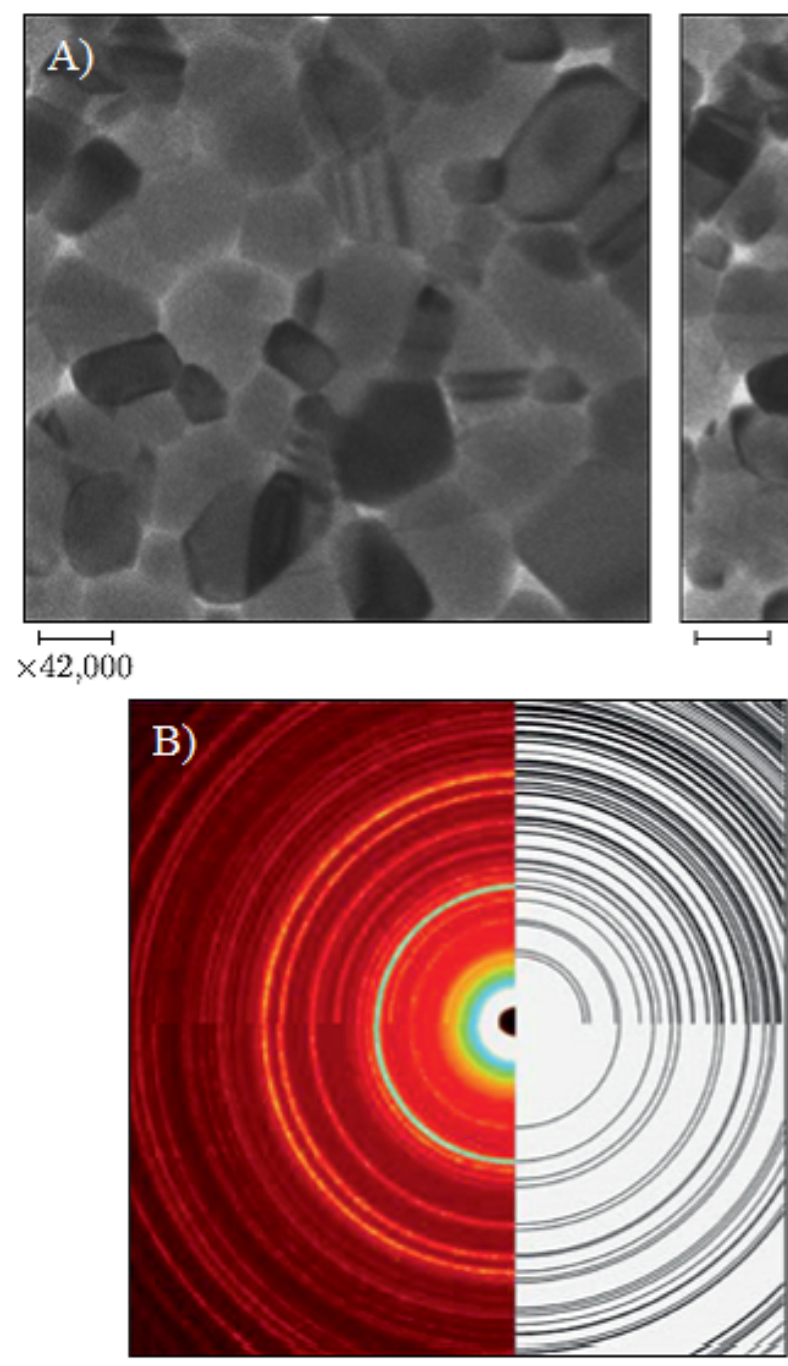

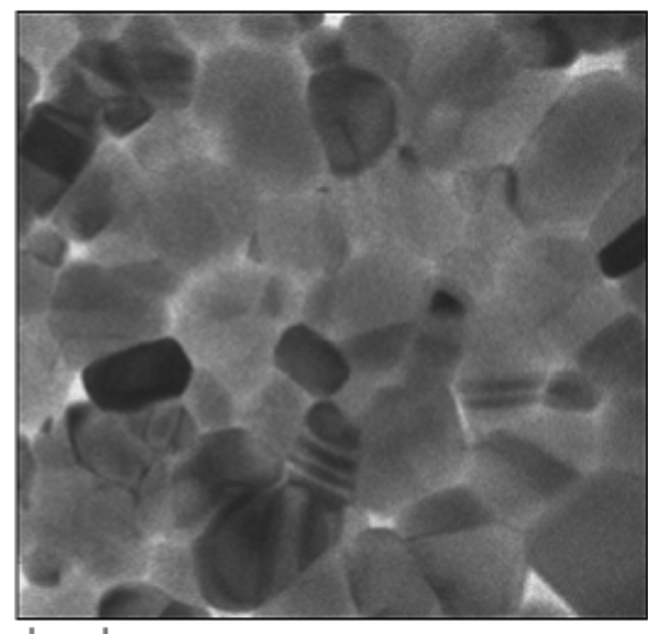

M
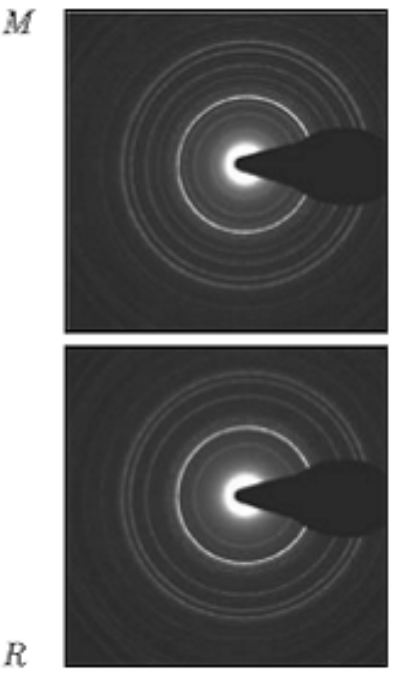

Figure 2. (A) Images obtained by the ultrafast electron microscopy (UEM) method before the phase transition in $\mathrm{VO}_{2}$ films (left) and after the phase transition (right). The magnification is $42,000 \times$ (scale $100 \mathrm{~nm}$ ). It should be noted that these images would not be observed if the femtosecond pulses for the generation of photoelectrons were blocked. (B) Diffraction patterns obtained by the UEM method before the phase transition in $\mathrm{VO}_{2}$ (right) and after it (left). Diffraction patterns of the two phases (monoclinic phase $\mathbf{M}$ and high-temperature tetragonal rutile phase $\mathbf{R}$ ) observed experimentally (on the left in panel B) and constructed as a result of calculations (on the right in panel B). The analysis was described in [38]. (Adapted from work in [38] with minor changes. Copyright (2006) National Academy of Sciences.)

The detection of the laser-induced metal-insulator transition in this material was the first example of an ultrafast structural phase transition that was visualized by the UEM method. Later, the structural dynamics of this process was examined by the UED method, when pulses containing up to $10^{7}$ electrons were used to probe coherent phenomena in $\mathrm{VO}_{2}$ in combination with ultrafast IR laser spectroscopy $[33,42]$. 


\subsection{UEM Imaging of Electromagnetic Waveforms}

Investigation of interaction processes of electromagnetic radiation with matter on their natural spatiotemporal scales plays an important role for the development of photonics. Rapidly oscillating electromagnetic fields underlie the operation of almost any photonic or electronic device, for which an understanding of the dynamics of electrons and the spatiotemporal evolution of electromagnetic fields inside matter is the key to optimizing their performance.

In [43], it was demonstrated that the UEM method can be used to measure the motion of collective charge carriers and electromagnetic fields with subcycle temporal and subwavelength spatial resolution (Figure 3). As a sample, a metal resonator with a ring radius of $230 \mu \mathrm{m}$ was taken, which can be used as a metamaterial. The sample was excited by single-cycle terahertz pulses in a $0.1-0.8 \mathrm{THz}$ spectral window, which were prepared using femtosecond laser radiation. The electromagnetic excitation in the metamaterial was detected by a pulsed electron beam with a kinetic energy of $70 \mathrm{keV}$ and a pulse repetition rate of $50 \mathrm{kHz}$. In the experiment, the compression of electronic pulses was provided by a laser-induced terahertz field that was superimposed with an electron beam during the passage through a very thin metal foil. As a result, the duration of the probe pulses in the plane of the sample, of $80 \mathrm{fs}$, turned out to be an order of magnitude shorter than the half-period of the exciting electromagnetic field, which ensured conditions for the detection of electrodynamic phenomena in the sample with high spatiotemporal resolution.

\subsection{Combining UEM with EELS}

In the course of ultrafast electron microscopy development, a completely new field of research of optical responses from nanostructures has been discovered, which is based on the interaction between a pulsed electron beam and radiation of a femtosecond laser. The authors of [44] presented the so-called photon-induced near-field electron microscopy (PINEM), which provides femtosecond temporal and nanometer spatial resolution. As an electron bunch passes through a region with an electromagnetic field, one part of the electrons in this pulse slows down, while the other part accelerates depending on when they flew into and out of this region. Precision measurements showed that changes in the kinetic energy correspond to an integer number of the energy of photons in the electromagnetic field. This effect depends on the intensity of the electromagnetic field, which makes it possible to visualize the distribution of the light field in the vicinity of a nanostructure by the PINEM method.

As a typical example, we will present experimental data that were obtained when a carbon nanotube $(\mathrm{CN})$ was irradiated by ultrashort laser pump pulses and was probed with a pulsed electron beam. To experimentally apply the method of EELS, a precision measurement of electron energy was carried out [44]. Thus, it was found that if electrons fly through the vicinity of the $\mathrm{CN}$ a few picoseconds before the laser pulse, then the electron energy experiences no changes. However, if electrons and light are incident onto the sample simultaneously, symmetrical side bands appear on the energy spectrum on both sides of the EELS peak, which corresponds to zero losses. The use of the electron beam energy filtering made it possible to obtain a near-field image in the vicinity of the $\mathrm{CN}$ in real time [44].

The spatial resolution of $6 \mathrm{~nm}$ in the PINEM scheme was achieved in an experiment with a silver sample [45]. In that work, a triangular Ag nanostructure with a characteristic length of $130 \mathrm{~nm}$ and a thickness of $20 \mathrm{~nm}$ was placed on a graphene substrate (Figure 4) and was irradiated with femtosecond laser pulses at a wavelength of $520 \mathrm{~nm}$ to excite plasmons in the sample. The sample was probed by a beam of ultrashort electron pulses with a diameter of $10 \mathrm{~nm}$ in the regime of scanning microscopy. A change in the electron energy was detected in the space-time continuum at different positions of the probe with respect to the sample and different time delays between the pump and probe pulses. 


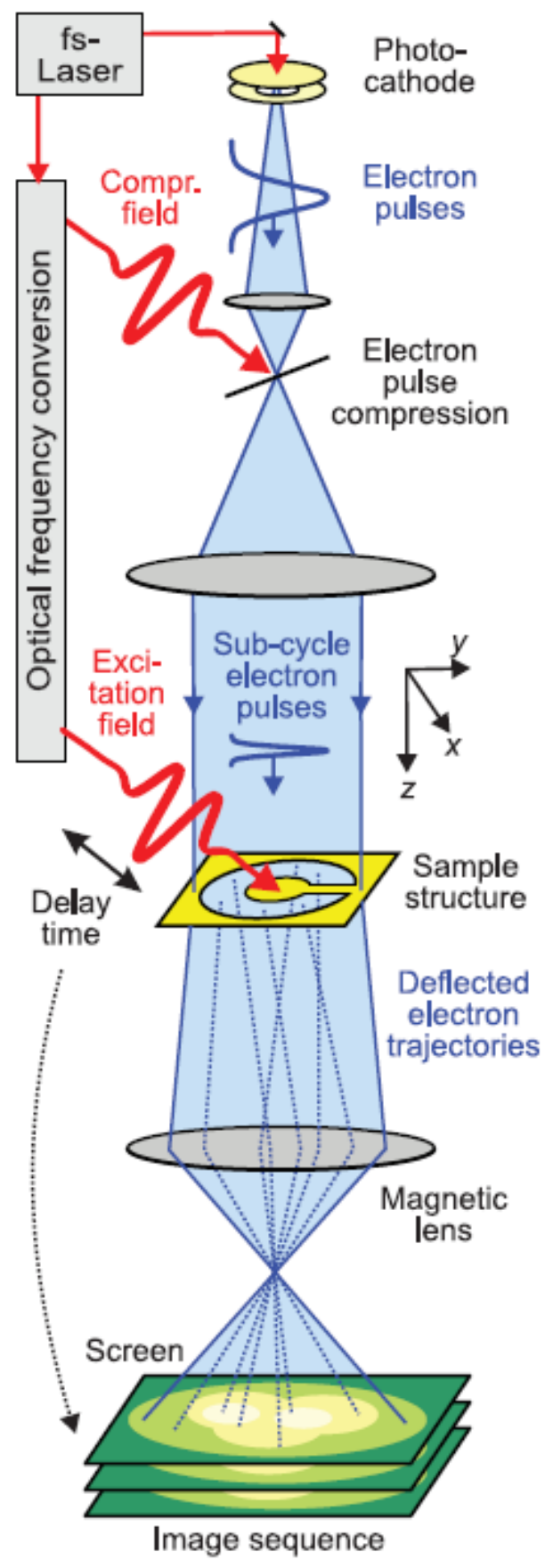

Figure 3. Schematic diagram of electron microscopy of electromagnetic waves [43]. Some part of the femtosecond laser radiation irradiated the photocathode to generate a pulsed electron beam, while the rest formed single-cycle terahertz pulses. The terahertz radiation, in turn, compressed electron pulses, and, also, induced electromagnetic resonance in the sample. As a result, the pulsed electron beam of femtosecond duration collimated by magnetic lenses passed through a metamagnetic resonator excited by single-cycle electromagnetic bursts. Because the probe pulses were shorter than the half-period of the electromagnetic field, the Lorentz forces "frozen" in time distorted the image of the excited sample, thereby providing a unique opportunity to visualize the electromagnetic field inside the substance with detection of its phase, amplitude, and polarization. Figure adapted from work in [43] with permission from Science AAAS. 


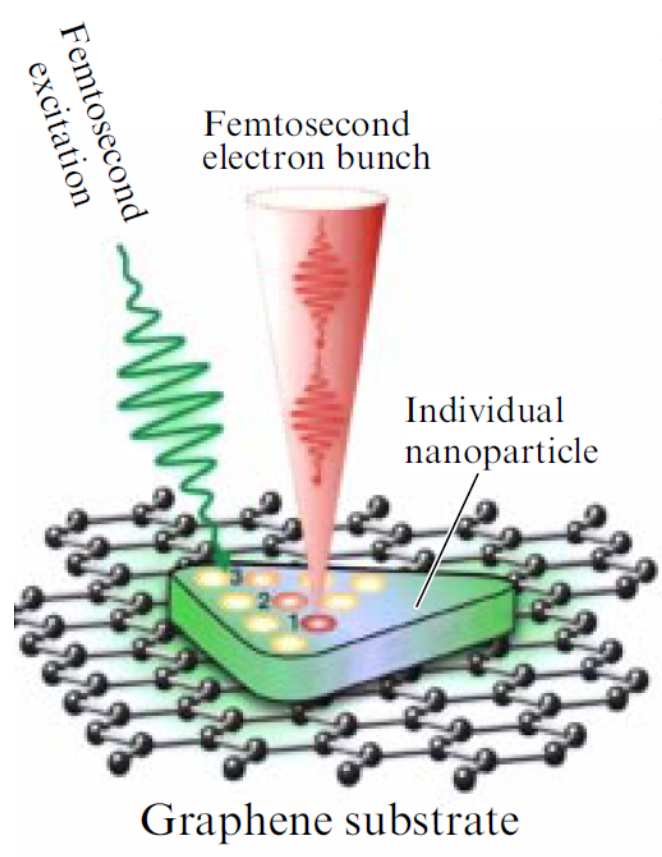

Shots demonstrating high spatial, temporal, and spectral (by energy) resolution

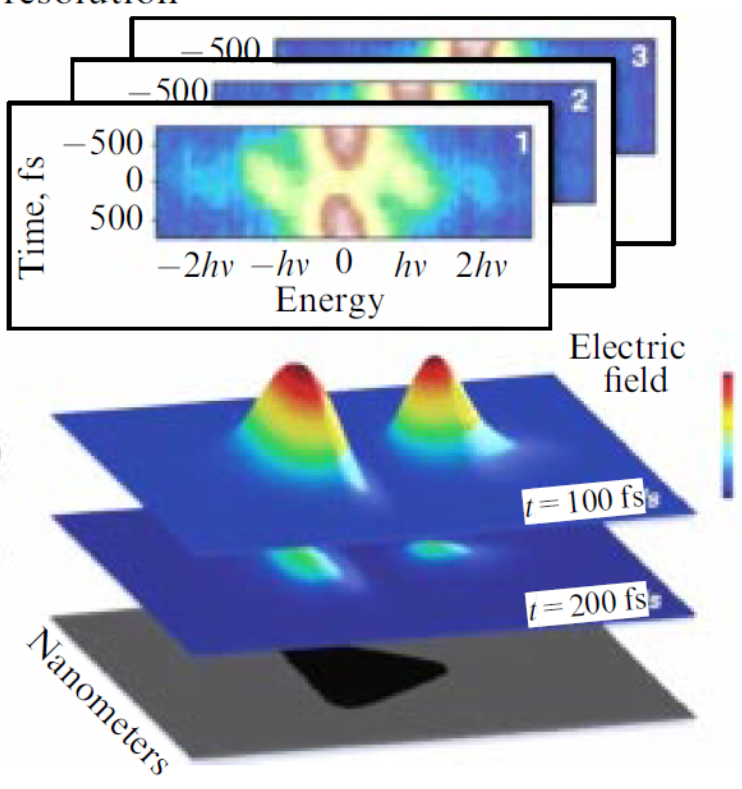

Figure 4. Subparticle ultrafast spectrum imaging in UEM. The change in the electron kinetic energy (electron spectrum) was measured as a function of the delay between an exciting femtosecond laser and probing electron pulses for each position of the probe. Energy gain was measured in units of laser radiation quantum, $h v=2.4 \mathrm{eV}$ [45]. Figure was adapted from work in [45] with permission from Science AAAS.

The UEM method in the EELS regime made it possible to observe the dynamics of chemical bonds in graphite when the sample was excited by femtosecond laser radiation [46]. In this experiment, a change in the electron energy loss spectrum was detected, which contained information on the plasmon properties of the material and made it possible to study the collective dynamics of valence electrons in graphite. The obtained experimental data reflected a laser-induced change in the electronic structure of the substance, consisting in hybridization of the sp2/sp3 type [46]. At present time, the PINEM method has received further development [47-51].

\subsection{Novel Trends in UEM: From 4D UEM to 5D UEM and to Attosecond Electron Microscopy}

The development of the UEM method made it possible to study laser-induced processes with high spatiotemporal resolution in the $4 \mathrm{D}$ continuum. An additional analysis of the electron energy in this method opened a new page in the detection of the optical response from a nanostructure, as a result of which it became experimentally possible to receive access to a fairly wide class of parameters characterizing the behavior of matter. Thus, prior to publishing experimental work [52], phonons and collective plasmon resonances in a sample could be imaged with atomic spatial and $10 \mathrm{meV}$ energy resolution using a conventional transmission electron microscope only under stationary conditions, which ruled out the observation of fast processes with high time resolution. At the same time, ultrafast all-optical spectroscopy was unable to provide the necessary spatial resolution. In [52], an ultrafast transmission electron microscope was used to detect laser-induced plasmons with an energy resolution of $20 \mathrm{meV}$, which was determined solely by the laser line width (Figure 5), for which the spectrally resolved PINEM method was used. As a result of the development of such an approach, an opportunity has opened up to study in detail various optically accessible phonons and collective plasmon resonances in matter with a high spatiotemporal resolution supplemented by energy resolution. 

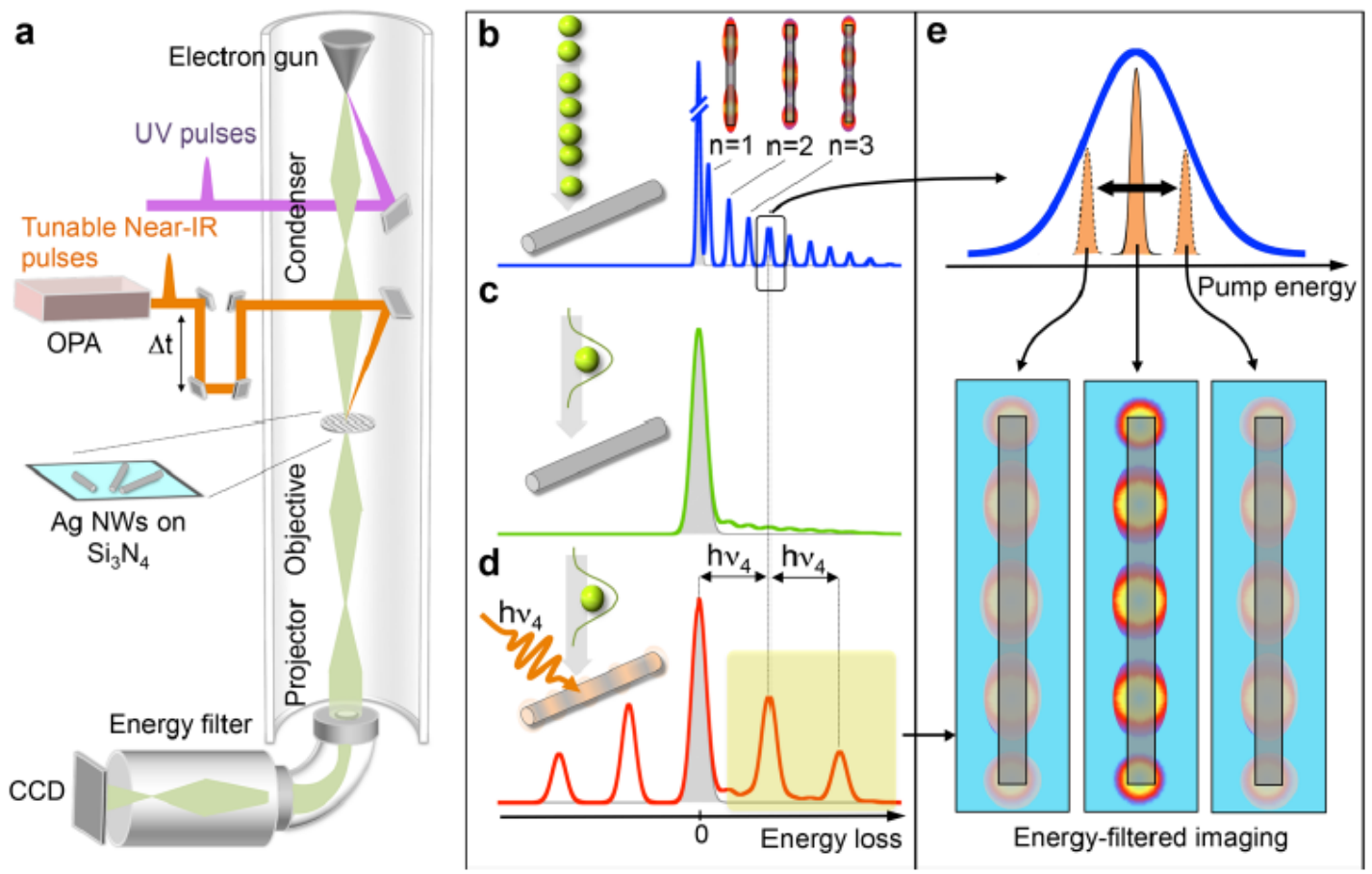

Figure 5. Spectrally resolved PINEN [52]. (a) Experimental scheme. (b) Electron energy-loss spectroscopy (EELS) spectrum revealing multiple plasmon resonances in an individual nanowire excited by passing electrons and mapped by raster scanning the electron beam (plasmon map insets). (c) Sketch of an electron energy loss (EEL) spectrum of the sample under ultrafast energy-filtered microscopy conditions. The visibility of electron-induced plasmon resonances is severely reduced. (d) Sketch of an EEL spectrum of the specimen (e.g., long silver nanowire (130 nm diameter, $7.8 \mu \mathrm{m}$ length) deposited on a $\mathrm{Si}_{3} \mathrm{~N}_{4}$ membrane) upon photoexcitation by light pulses (e.g., the pump with $100 \mathrm{fs}, 1.08 \mathrm{eV}$, and $5 \mathrm{~mJ} / \mathrm{cm}^{2}$ laser pulses from an optical parametric amplifier) of energy $h v_{4}$, tuned to the $n=4$ plasmon-resonance frequency. This specific mode exchanges several times its characteristic energy with electrons. (e) Concept of the experiment in [52]: the laser excitation wavelength is scanned and the plasmon resonance profile is retrieved via quantitative analysis of the energy-filtered images in UEM. Reprinted (adapted) with permission from work in [52]. Copyright (2018) American Chemical Society.

It was noted in [52] that such a method, which could be termed the "5D UEM," in contrast to traditional methods of inelastic scattering, was not limited to the energy broadening of the probe electron beam. In fact, the characteristic energy and the resolution necessary to display a particular optically accessible mode are determined solely by the spectral properties of the excitation channel, rather than by the characteristics of the electron beam or electron spectrometer in the EELS scheme, which is of particular interest for photonics and plasmonics. Indeed, the possibility of generating monochromatic laser radiation with a controlled time profile in the spectral range from UV to IR provides, within the framework of this approach, unprecedented access to a wider class of parameters compared to conventional electronic optics. The developed approach can be used to detect almost any low-energy modes in condensed matter, provided that they are selectively excited by laser radiation. In particular, by measuring spatiotemporal and spectral profiles related to laser-induced phonons in a solid substance, it will be possible to characterize the dynamic coupling between the lattice and the electronic subsystem, which opens up the possibility of controlling the dynamics of perspective materials. Moreover, such an approach, combined with the method of selective population of vibrational states in catalysts based on plasmon nanostructures, can provide important information on the trajectories of chemical reactions [52]. 
Formation of attosecond electron bursts is of undoubted interest for probing the ultrafast electron dynamics in matter [53-57]. However, the time resolution of the "classical" UEM experimental setups is limited by the initial duration of the photoelectron bunch at the exit out from the accelerating gap, $\sim 100 \mathrm{fs}$, and the Coulomb repulsion effect [1,4], which significantly impedes and, in the general case, does not allow direct detection of the electronic dynamics. An optical gating method using intense femtosecond laser radiation was demonstrated in [58], when free electrons exchange energy with photons, which made it possible to prepare $30 \mathrm{fs}$ electron pulses. In this method, an intense ultrashort laser pulse serves as an ultrafast gate for the electron beam, which leads to a discrete change in the kinetic energy of electrons when the peaks in the energy space are separated by one or more quanta of the optical gate. After filtering the electron beam by energy, it becomes possible to form electron bursts with a time profile similar to that of the optical gate pulse. To control electrons on a subfemtosecond time scale, pulsed optical radiation of attosecond duration can be used as a gate, which was demonstrated previously in [59]. As a result of this approach, which is illustrated in Figure 6, isolated attosecond electron pulses can be created to directly detect ultrafast electronic dynamics in atomic-molecular structures and condensed matter with the necessary spatiotemporal resolution.

\subsection{Holographic Imaging of Electromagnetic Fields via Electron-Light Quantum Interference}

Two recent articles $[60,61]$ have been devoted to the creation of holograms of a fundamentally new type based on the scattering of a pulsed electron beam in a strong laser field. As is well known, the fundamental principle of holography is to record the phase and the amplitude of the distribution of a signal, mixing it with a known reference signal. This brilliant idea was proposed by Gabor to improve the spatial resolution of an electron microscope, but, originally, it was applied primarily in light optics. Application of a similar concept to time-resolved measurements should provide an unprecedented ultrahigh spatiotemporal resolution. In the UEM experiment in [60], it was shown that, by creating holograms of local electromagnetic fields, attosecond resolution can be achieved in combination with nanometer resolution (Figure 7). As distinct from classical optical holography, where the signal and reference fields are initially spatially separated and then are mixed to create an interference pattern in the image plane, the new approach demonstrated in [60] is based on electromagnetic fields that split the electron wave function into a quantum coherent superposition of different energy states. As a result, the spatial modulation of the electron energy distribution reflects the phase relationship between the reference and signal fields. The authors of $[60,61]$ also discussed the application of such an approach for electron quantum optics in the framework of parallel quantum measurements.

Here, we can recall that, in the extreme case, to ensure ultrahigh spatiotemporal resolution, not limited by Coulomb repulsion, the UEM setup actually deals with single-electron wave packets [62]. However, as far as we know, their correct description, in contrast to the multielectron case, faces serious conceptual difficulties. In this regard, we can formulate the following question, which we leave unanswered. Is it possible to expect the appearance of some new unusual properties of single-electron wave packets if an ultrafast transmission electron microscope is used as a test bench? In this connection, we note that the identities of a new type that were reported in theoretical paper [63] make it possible to convert the densities of local pseudo-pulses into a local pulse density when analyzing the dynamics of electron wave packets, which greatly simplifies their description. 


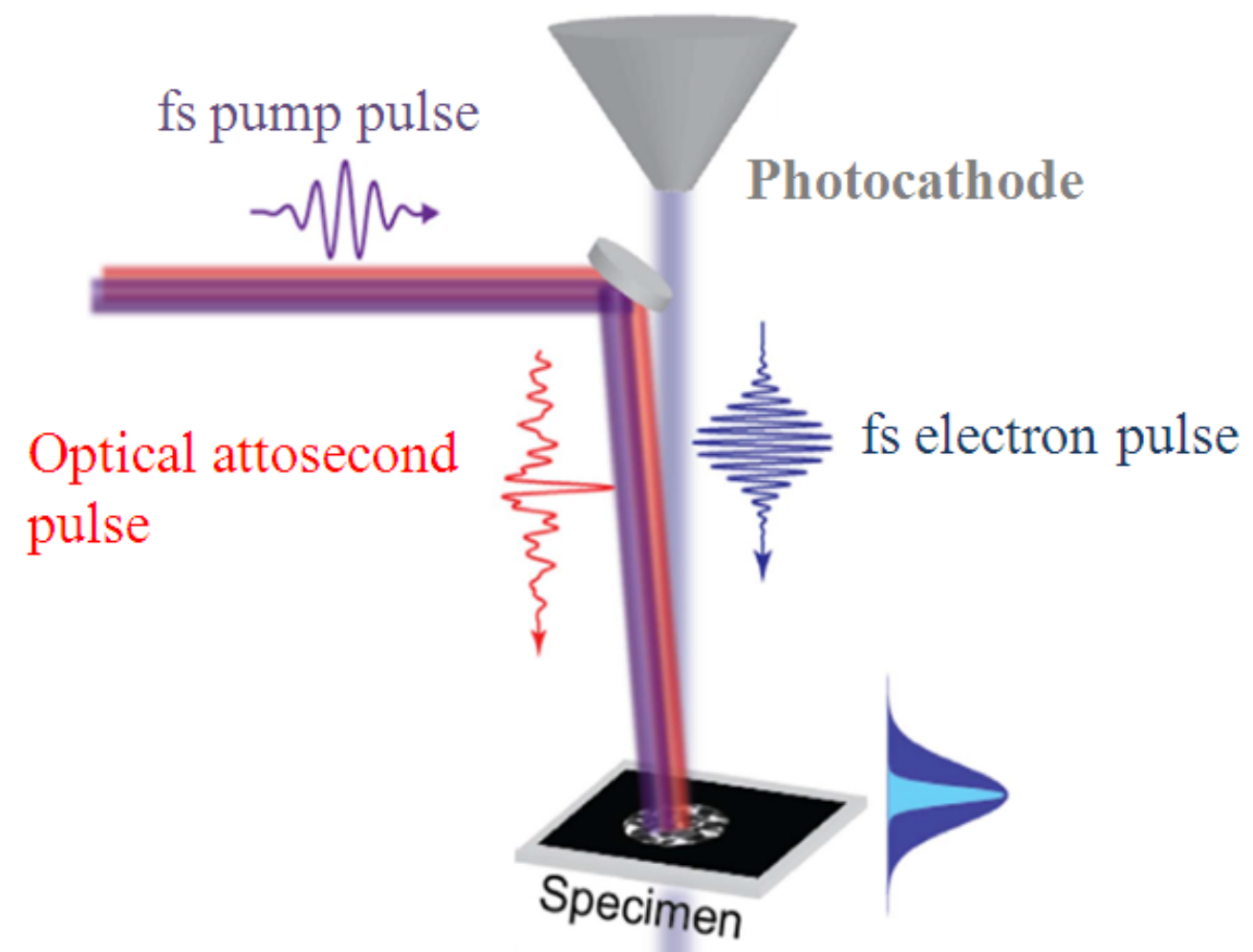

Energy analyser

Sub-fs gated electron pulse

Figure 6. Illustration explaining the conceptual scheme of attosecond electron microscopy. Here, an optical attosecond pulse serves as an instantaneous gate to form an isolated ultrashort electron burst, which should be filtered after gating. As a result, the prepared attosecond free electron pulses can be used to detect ultrafast laser-induced electron dynamics in real-time (adapted from work in [54] with minor changes). 


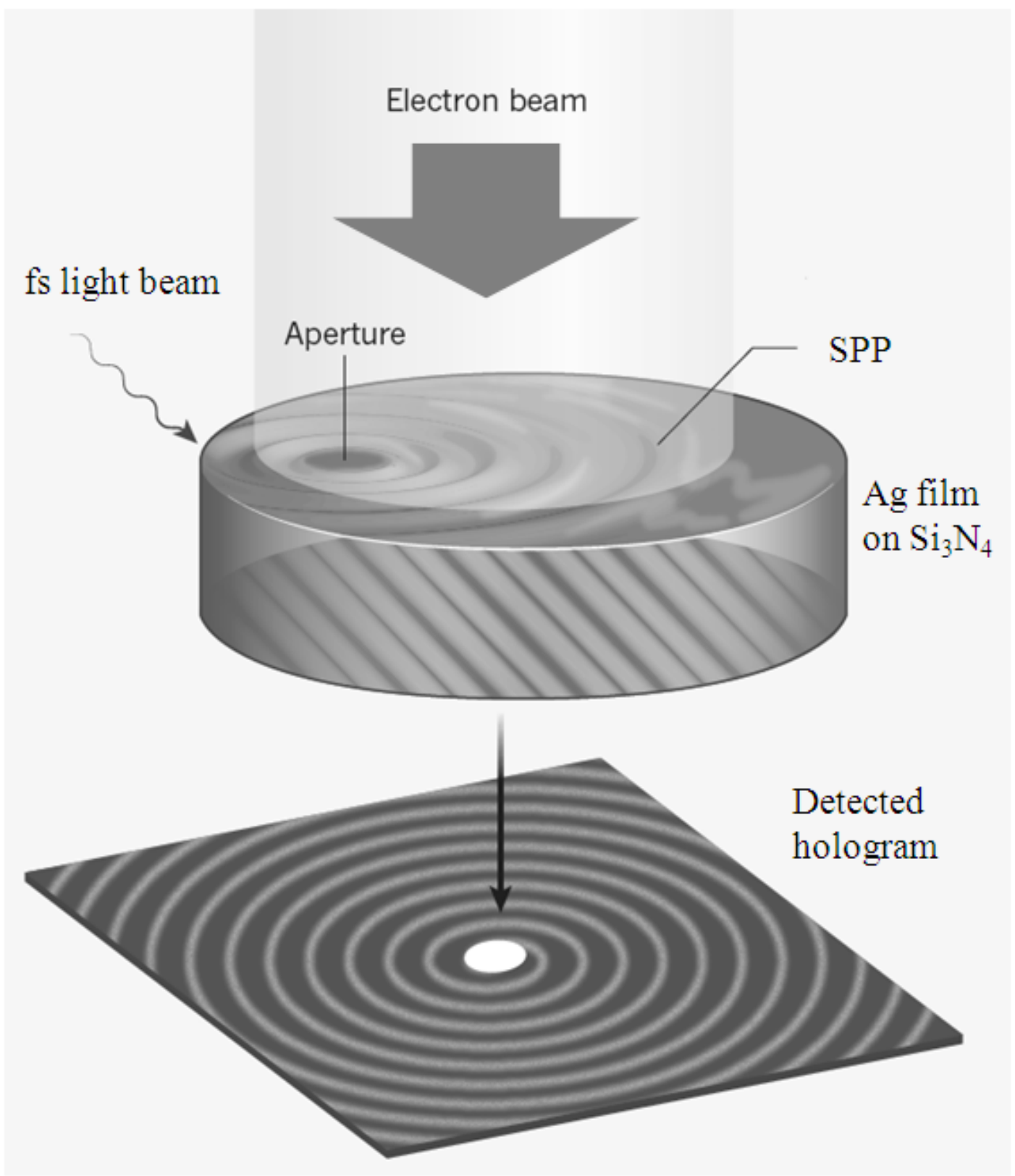

Figure 7. Ultrafast mapping of electron-light interference: In the experiment in [60], light irradiates a metal film that contains an aperture, to produce surface plasmon polaritons (SPPs). A different light-field pattern, which is illustrated by stripes, is produced on the other side of the film. When an electron beam passes through the specimen, it subsequently interacts with the fields on both sides, producing a spiral interference pattern. This pattern encodes the relative phases of the light fields at each position on the film, and therefore contains holographic information (adapted from [61] with minor changes).

\subsection{UEM with Laser-Driven Nanoemitters}

Conventional transmission electron microscopes use three types of cathodes, which are based on the thermionic principle, on the Schottky emitter, and on the cold-field emission. In the latter case, no heating of the cathode is required, and the extracting electric field is applied to the sharp conical metal tip. In turn, to generate a continuous electron beam, the Schottky cathode operates in the regime of thermionic emission amplified in a strong electric field. 
Ultrafast transmission electron microscopes equipped with flat emitters fail to provide high brightness due to a relatively large size of the photoelectron source, which limits possible applications of UEM, including electron holography. The use of nanoemitters opens a new page in the development of this field of research.

An ultrafast transmission electron microscope, in which an electron beam is formed as a result of nanolocal laser-stimulated emission from a Schottky cathode, was first demonstrated in [64]. In this instrument, a JEOL JEM-2100F transmission electron microscope with kinetic beam energy of up to $200 \mathrm{keV}$ was used, which was modified to study laser-induced processes with high temporal resolution. $\mathrm{A} \mathrm{ZrO} / \mathrm{W}$-based photocathode with a tip diameter of less than $100 \mathrm{~nm}$ was irradiated with the second harmonic of a femtosecond Ti:Sa laser with a wavelength of $400 \mathrm{~nm}$ and a pulse repetition rate of up to $800 \mathrm{kHz}$. In a strong static extracting electric field, reaching a value of $1 \mathrm{~V} / \mathrm{nm}$, the cathode work function lowered to $2.9 \mathrm{eV}$, which ensured the condition of single-photon electron emission. It is also important to note that, in the stationary regime, such a cathode was heated to a temperature of $1800 \mathrm{~K}$. In the photoemission regime, the heating lowered to a temperature of $1400 \mathrm{~K}$, which completely ruled out the possibility of thermal emission. As a result, unprecedented UEM characteristics were achieved with a focused electron beam diameter of down to $9 \AA$ and an electron pulse duration of down to $200 \mathrm{fs}$. The energy spread in the electron beam was $\sim 0.6 \mathrm{eV}$. The electronic emission localized at the cathode tip is accompanied by minimization of the phase space volume occupied by photoelectrons, which, in turn, ensures high coherence of the beam. Thus, in the experiment of [64], the transverse coherence of about $1.2 \mu \mathrm{m}$ was achieved. The created experimental setup can operate both in the imaging and in the electron diffraction modes with a high temporal resolution. The possibility of using coherent quantum interactions between a strong laser field and free electrons in the beam was also discussed. Potential applications of the developed method include holography and electron spectroscopy. It was noted that this experimental configuration allows operating in a wide time range from continuous to femtosecond regime.

In another work [65], to develop the UEM method, a cold-field emitter based on a tungsten tip with a characteristic size of the apex of just over $100 \mathrm{~nm}$ was used, which resulted in an unprecedented high brightness for an ultrafast electron microscope. For this purpose, a Hitachi High Technologies (HHT) HF2000 transmission electron microscope with electron energies of up to $200 \mathrm{keV}$ was adapted to UEM requirements. In the experiment, the photocathode was irradiated with a tightly focused second harmonic of a femtosecond fiber laser at a wavelength of $515 \mathrm{~nm}$ and a pulse repetition rate of up to $1 \mathrm{MHz}$. In contrast to work in [64], a multiphoton regime of electron emission was realized here. Thus, at a laser pulse energy of about $5 \mathrm{~nJ}$, each bunch contained roughly 20 photoelectrons, which made it possible to perform electron holography in an off-axis configuration.

\subsection{Ultrafast Lorentz Microscopy}

The authors of [66] developed the experimental foundations of Femtosecond Lorentz Microscopy to study fast processes associated with spin dynamics in matter. In Lorentz transmission electron microscopy, the Lorentz force deflects electrons in the beam propagating orthogonally to the magnetic field. With respect to the visualization scheme, Lorentz microscopy is divided into Fresnel microscopy and Foucault microscopy. In the first case, narrow areas of relatively high and low intensities are formed on the screen in positions that correspond to the domain walls, which is caused by small deflections of electrons passing through the sample. Depending on the direction of magnetization of each domain, the Lorentz force will slightly deflect the electron beam either to one wall of the domain or to the other, as a result of which the image of the domain will have a bright wall on one side and dark on the other. In the second case, the image remains to be focused, and a diffraction pattern is formed in one of the planes of the transmission microscope. By displacing the diaphragm, one can block electrons that have been deflected in one direction by the magnetic field. In this case, the brightness of each domain on the screen depends on the direction of its magnetization. In [66], Fresnel femtosecond microscopy was implemented. 
In the demonstration experiment, a laser-induced demagnetization process of a single magnetic vortex structure was imaged in the space-time continuum with determining the corresponding magnetization field. In the experiment, an isolated permalloy disk with a diameter of $1 \mu \mathrm{m}$ and a thickness of $20 \mathrm{~nm}$ preliminarily deposited onto a $50 \mathrm{~nm}$ silicon nitride membrane was irradiated with $50 \mathrm{fs}$ laser pulses at a wavelength of $800 \mathrm{~nm}$ with a repetition frequency of $500 \mathrm{kHz}$. The energy density in the pulse reached a few $\mathrm{mJ} / \mathrm{cm}^{2}$, up to $8.5 \mathrm{~mJ} / \mathrm{cm}^{2}$, for the optical excitation of the magnetic vortex structure. In a permalloy film, the magnetization vector is orthogonal to the sample surface. As a result of irradiation of the material with powerful laser pulses, a component of the magnetic field along the surface of the film appeared.

The spatial distribution of the sample magnetization was detected in the regime of transmission electron microscopy using single electron femtosecond bunches with a high repetition rate, which were obtained by means of a nanolocalized laser-induced emission process, which ensured a high spatial coherence of up to $\sim 1 \mu \mathrm{m}$. In the experiment, sub-100 $\mathrm{nm}$ spatial and $700 \mathrm{fs}$ time resolution were achieved. This method of imaging of the ultrafast dynamics of magnetization of matter is of particular value for the optical control of magnetic nanoscale structures [67]. The development of this approach opens up the possibility of imaging of ultrafast internal dynamics of magneto-topological defects on a scale of $10 \mathrm{~nm}$.

\section{Conclusions}

The rapidly developing methods of ultrafast electron microscopy and diffraction make it possible to monitor fast laser-induced processes in matter on their natural spatiotemporal scales using ultrashort electron pulses. Detection of atomic-molecular structures in the space-time continuum opens up the possibility of making a molecular movie, which requires ultrahigh temporal and spatial resolution along with high measurement sensitivity. Comparing the two approaches, we should note that electron diffraction is a considerably more sensitive method than real-space mapping. As applied to developed pulsed electron sources, this makes UED actually the only possible method to detect the atomic-molecular structure per one probing pulse. This method makes it possible to ensure a femtosecond time resolution even if each probe pulse contains $\sim 10^{6}-10^{7}$ electrons, which is achieved by using a cavity with an RF field and using an extremely compact scheme in which the cathode-sample distance is reduced to a minimum. An alternative approach is the passage to relativistic velocities, or the development of other methods of compression of electron pulses, which opens up the possibility of observing irreversible fast processes in complex molecular systems.

The advantage of the UEM method is its possibility to work in several modes. Apart from observation of electron diffraction, this method makes it possible to detect ultrafast processes in matter in the real-space mapping, when it becomes possible to experimentally visualize spatial inhomogeneities of the sample, as well as to determine their local effect on the structural dynamics. High-precision measurements of the kinetic energy of electrons in the transmission microscope column using EELS significantly extend the capabilities of UEM, which, on the one hand, allows one to pass in the perspective to the 5D UEM technique for imaging the fast dynamics with nanometer spatial, femtosecond temporal, and $~ 1-\mathrm{meV}$ energy resolution; on the other hand, attosecond microscopy can be developed on this basis to detect the electron dynamics in matter. The use of a nanoemitter as a photocathode allows one to provide high brightness and to achieve high spatial coherence, $\sim 1 \mu \mathrm{m}$ in the UEM method. Such a photoelectron source was used in ultrafast Lorentz microscopy, which opened up the possibility of studying the ultrafast dynamics of magnetization of matter within the framework of the optical control of magnetic nanosized structures.

In this article, to demonstrate the potential of UED, we presented a number of experiments that were carried out in recent years after the publication of the review [5]. In the method of time-resolved transmission electron microscopy [11], the UEM approach was distinguished, which provides ultrahigh spatiotemporal resolution. Here, we confined ourselves to the illustration of possibilities of this method using examples of direct observation of laser-induced ultrafast dynamics in matter. 
Funding: This work was supported by the Russian Foundation for Basic Research (Grant No. 20-02-00146 A).

Conflicts of Interest: The authors declare no conflict of interest.

\section{References}

1. Zewail, A.H.; Thomas, J.M. 4D Electron Microscopy. Imaging in Space and Time; Imperial College Press: London, UK, 2010.

2. Chergui, M.; Zewail, A.H. Electron and X-ray methods of ultrafast structural dynamics: Advances and applications. ChemPhysChem 2009, 10, 28-43. [CrossRef]

3. Miller, R.J.D. Mapping Atomic Motions with Ultra-bright Electrons: The Chemists' Gedanken Experiment Enters the Lab Frame. Annu. Rev. Phys. Chem. 2014, 65, 583-604. [CrossRef] [PubMed]

4. Ischenko, A.A.; Aseyev, S.A. Time Resolved Electron Diffraction: For Chemistry, Biology and Materials Science; Elsevier: San Diego, CA, USA, 2014.

5. Ischenko, A.A.; Weber, P.M.; Miller, R.J.D. Capturing Chemistry in Action with Electrons: Realization of Atomically Resolved Reaction Dynamics. Chem. Rev. 2017, 117, 11066-11124. [CrossRef] [PubMed]

6. Ischenko, A.A.; Golubkov, V.V.; Spiridonov, V.P.; Zgurskii, A.V.; Akhmanov, A.S.; Vabischevich, M.G.; Bagratashvili, V.N. A stroboscopical gas-electron diffraction method for the investigation of short-lived molecular species. Appl. Phys. B 1983, 32, 161-163. [CrossRef]

7. Siwick, B.J.; Dwyer, J.R.; Jordan, R.E.; Miller, R.J.D. An Atomic-Level View of Melting Using Femtosecond Electron Diffraction. Science 2003, 302, 1382-1385. [CrossRef]

8. Siwick, B.J.; Dwyer, J.R.; Jordan, R.E.; Miller, R.J.D. Ultrafast Electron Optics: Propagation Dynamics of Femtosecond Electron Packets. J. Appl. Phys. 2002, 92, 1643-1648. [CrossRef]

9. Muller, D.A. Structure and bonding at the atomic scale by scanning transmission electron microscopy. Nat. Mater. 2009, 8, 263-270. [CrossRef]

10. Uhlemann, S.; Müller, H.; Hartel, P.; Zach, J.; Haider, M. Thermal Magnetic Field Noise Limits Resolution in Transmission Electron Microscopy. Phys. Rev. Lett. 2013, 111, 046101. [CrossRef]

11. Campbell, G.H.; McKeown, J.T.; Santala, M.K. High-Speed Electron Microscopy. In Springer Handbook of Microscopy; Springer Handbooks; Hawkes, P.W., Spence, J.C.H., Eds.; Springer Nature AG: Gewerbestrasse, Cham, Switzerland, 2019; pp. 455-485.

12. King, W.E.; Campbell, G.H.; Frank, A.; Reed, B.; Schmerge, J.F.; Siwick, B.J.; Stuart, B.C.; Weber, P.M. Ultrafast electron microscopy in materials science, biology, and chemistry. J. Appl. Phys. 2005, 97, 111101. [CrossRef]

13. Van Oudheusden, T.; De Jong, E.F.; Van der Geer, S.B.; Op't Root, W.P.E.M.; Luiten, O.J.; Siwick, B.J. Electron Source Concept for Single-Shot Sub-100 Fs Electron Diffraction in the $100 \mathrm{keV}$ Range. J. Appl. Phys. 2007, 102, 093501. [CrossRef]

14. Weber, P.M.; Carpenter, S.D.; Lucza, T. A reflectron design for femtosecond electron guns. SPIE 1995, 2521, 23-29.

15. Mankos, M.; Shadman, K.; Siwick, B.J. A novel electron mirror pulse compressor. Ultramicroscopy 2017, 183, 77-83. [CrossRef]

16. Waldecker, L.; Bertoni, R.; Ernstorfer, R. Compact Femtosecond Electron Diffractometer with 100 keV Electron Bunches Approaching the Single-Electron Pulse Duration Limit. J. Appl. Phys. 2015, 117, 044903. [CrossRef]

17. Jinfeng, Y.; Kazuki, G.; Nobuyasu, N.; Shouichi, S.; Yoichi, Y. A Compact Ultrafast Electron Diffractometer with Relativistic Femtosecond Electron Pulses. Quantum Beam Sci. 2020, 4, 4.

18. Hastings, J.B.; Rudakov, F.M.; Dowell, D.H.; Schmerge, J.F.; Cardoza, J.D.; Castro, J.M.; Gierman, S.M.; Loos, H.; Weber, P.M. Ultrafast Time-Resolved Electron Diffraction with Megavolt Electron Beams. Appl. Phys. Lett. 2006, 89, 184109. [CrossRef]

19. Van Oudheusden, T.; Pasmans, P.L.E.M.; Van der Geer, S.B.; De Loos, M.J.; Van der Wiel, M.J.; Luiten, O.J. Compression of Subrelativistic Space-Charge-Dominated Electron Bunches for Single-Shot Femtosecond Electron Diffraction. Phys. Rev. Lett. 2010, 105, 264801. [CrossRef]

20. Hada, M.; Miyata, K.; Ohmura, S.; Arashida, Y.; Ichiyanagi, K.; Katayama, I.; Suzuki, T.; Chen, W.; Mizote, S.; Sawa, T.; et al. Selective Reduction Mechanism of Graphene Oxide Driven by the Photon Mode versus the Thermal Mode. ACS Nano 2019, 13, 10103-10112. [CrossRef] 
21. Hada, M.; Yamaguchi, D.; Ishikawa, T.; Sawa, T.; Tsuruta, K.; Ishikawa, K.; Koshihara, S.; Hayashi, Y.; Kato, T. Ultrafast isomerization-induced cooperative motions to higher molecular orientation in smectic liquid-crystalline azobenzene molecules. Nat. Commun. 2019, 10, 4159. [CrossRef]

22. Shen, X.; Nunes, J.P.F.; Yang, J.; Jobe, R.K.; Li, R.K.; Lin, M.-F.; Moore, B.; Niebuhr, M.; Weathersby, S.P.; Wolf, T.J.A.; et al. Femtosecond gas-phase mega-electron-volt ultrafast electron diffraction. Struct. Dyn. 2019, 6, 054305. [CrossRef]

23. Yang, J.; Zhu, X.; Wolf, T.J.A.; Li, Z.; Nunes, J.P.F.; Coffee, R.; Cryan, J.P.; Gühr, M.; Hegazy, K.; Heinz, T.F.; et al. Imaging $\mathrm{CF}_{3} \mathrm{I}$ conical intersection and photodissociation dynamics with ultrafast electron diffraction. Science 2018, 361, 64-67. [CrossRef]

24. Wolf, T.J.A.; Sanchez, D.M.; Yang, J.; Parrish, R.M.; Nunes, J.P.F.; Centurion, M.; Coffee, R.; Cryan, J.P.; Gühr, M.; Hegazy, K.; et al. The photochemical ring-opening of 1,3-cyclohexadiene imaged by ultrafast electron diffraction. Nat. Chem. 2019, 11, 504-509. [CrossRef] [PubMed]

25. Shin, T.; Wolfson, J.W.; Teitelbaum, S.W.; Kandyla, M.; Nelson, K.A. Carrier confinement and bond softening in photoexcited bismuth films. Phys. Rev. B 2015, 92, 184302. [CrossRef]

26. Rogacheva, E.I.; Grigorov, S.N.; Nashchekina, O.N.; Lyubchenko, S.; Dresselhaus, M.S. Quantum-size effects in n-type bismuth thin films. Appl. Phys. Lett. 2003, 82, 2628-2630. [CrossRef]

27. Rogacheva, E.I.; Lyubchenko, S.G.; Dresselhaus, M.S. Semimetal-semiconductor transition in thin Bi films. Thin Solid Films 2008, 516, 3411-3415. [CrossRef]

28. Bugayev, A.; Elsayed-Ali, H.E. Lattice dynamics and electronic Grüneisen parameters of femtosecond laser-excited bismuth. J. Phys. Chem. Solids 2019, 129, 312-316. [CrossRef]

29. Tinnemann, V.; Streubühr, C.; Hafke, B.; Kalus, A.; Hanisch-Blicharski, A.; Ligges, M.; Zhou, P.; von der Linde, D.; Bovensiepen, U.; Horn-von Hoegen, M. Ultrafast electron diffraction from a Bi(111) surface: Impulsive lattice excitation and Debye-Waller analysis at large momentum transfer. Struct. Dyn. 2019, 6, 035101. [CrossRef]

30. Tinnemann, V.; Streubühr, C.; Hafke, B.; Witte, T.; Kalus, A.; Hanisch-Blicharski, A.; Ligges, M.; Zhou, P.; von der Linde, D.; Bovensiepen, U.; et al. Decelerated lattice excitation and absence of bulk phonon modes at surfaces: Ultra-fast electron diffraction from Bi(111) surface upon fs-laser excitation. Struct. Dyn. 2019, 6, 065101. [CrossRef]

31. Jiang, Y.; Liu, L.C.; Sarracini, A.; Krawczyk, K.M.; Wentzell, J.S.; Lu, C.; Field, R.L.; Matar, S.F.; Gawelda, W.; Müller-Werkmeister, H.M.; et al. Direct observation of nuclear reorganization driven by ultrafast spin transitions. Nat. Commun. 2020, 11, 1530. [CrossRef]

32. De Cotret, L.P.R.; Pöhls, J.-H.; Stern, M.J.; Otto, M.R.; Sutton, M.; Siwick, B.J. Time- and momentum-resolved phonon population dynamics with ultrafast electron diffuse scattering. Phys. Rev. B 2019, 100, 214115. [CrossRef]

33. Otto, M.R.; De Cotret, L.P.R.; Valverde-Chavez, D.A.; Tiwari, K.L.; 'Emond, N.; Chaker, M.; Cooke, D.G.; Siwick, B.J. How optical excitation controls the structure and properties of vanadium dioxide. Proc. Natl. Acad. Sci. USA 2019, 116, 450-455. [CrossRef]

34. Otto, M.R.; Pöhls, J.-H.; de Cotret, L.P.R.; Stern, M.J.; Sutton, M.; Siwick, B.J. Ultrafast signatures of exciton-phonon coupling in TiSe 2 . arXiv 2019, arXiv:1912.03559.

35. Jing, C.; Zhu, Y.; Liu, A.; Schliep, K.; Fu, X.; Zhao, Y.; Montgomery, E.; Rush, W.; Kanareykin, A.; Katz, M.; et al. Tunable electron beam pulser for picoseconds stroboscopic microscopy in transmission electron microscopes. Ultramicroscopy 2019, 207, 112829. [CrossRef] [PubMed]

36. Spivak, G.V.; Saparin, G.V.; Bykov, M.V. Scanning electron microscopy. Sov. Phys. Usp. 1970, 12, 756-776. [CrossRef]

37. Bostanjoglo, O.; Rosin, T. Ultrasonically induced magnetic reversals observed by stroboscopic electron-microscopy. Opt. Acta 1977, 24, 657-664. [CrossRef]

38. Grinolds, M.S.; Lobastov, V.A.; Weissenrieder, J.; Zewail, A.H. Four-dimensional ultrafast electron microscopy of phase transitions. Proc. Natl. Acad. Sci. USA 2006, 103, 18427-18431. [CrossRef]

39. Cremons, D.R.; Plemmons, D.A.; Flannigan, D.J. Femtosecond electron imaging of defect-modulated phonon dynamics. Nat. Commun. 2016, 7, 11230. [CrossRef]

40. Cremons, D.R.; Du, D.X.; Flannigan, D.J. Picosecond phase-velocity dispersion of hypersonic phonons imaged with ultrafast electron microscopy. Phys. Rev. Mater. 2017, 1, 073801. [CrossRef] 
41. Zhang, Y.; Flannigan, D.J. Observation of Anisotropic Strain-Wave Dynamics and Few-Layer Dephasing in $\mathrm{MoS}_{2}$ with Ultrafast Electron Microscopy. Nano Lett. 2019, 19, 8216-8224. [CrossRef]

42. Morrison, V.R.; Chatelain, R.P.; Tiwari, K.L.; Hendaoui, A.; Bruhács, A.; Chaker, M.; Siwick, B.J. A photo-induced metal-like phase of monoclinic $\mathrm{VO}_{2}$ revealed by ultrafast electron diffraction. Science 2014, 346, 445-448. [CrossRef]

43. Ryabov, A.; Baum, P. Electron microscopy of electromagnetic waveforms. Science 2016, 353, $374-377$. [CrossRef]

44. Barwick, B.; Flannigan, D.J.; Zewail, A.H. Photon-induced near-field electron microscopy. Nature 2009, 462, 902-906. [CrossRef] [PubMed]

45. Yurtsever, A.; van der Veen, R.M.; Zewail, A.H. Subparticle ultrafast spectrum imaging in 4D electron microscopy. Science 2012, 335, 59-64. [CrossRef] [PubMed]

46. Carbone, F.; Kwon, O.-H.; Zewail, A.H. Dynamics of chemical bonding mapped by energy-resolved 4D electron microscopy. Science 2009, 325, 181-184. [CrossRef] [PubMed]

47. Lee, Y.M.; Kim, Y.J.; Kim, Y.J.; Kwon, O.H. Ultrafast electron microscopy integrated with a direct electron detection camera. Struct. Dynam. 2017, 4, 044023. [CrossRef]

48. Feist, A.; Echternkamp, K.E.; Schauss, J.; Yalunin, S.V.; Schafer, S.; Ropers, C. Quantum coherent optical phase modulation in an ultrafast transmission electron microscope. Nature 2015, 521, 200-203. [CrossRef]

49. Piazza, L.; Lummen, T.T.A.; Quinonez, E.; Murooka, Y.; Reed, B.W.; Barwick, B.; Carbone, F. Simultaneous observation of the quantization and the interference pattern of a plasmonic near-field. Nat. Commun. 2015, 6, 6407. [CrossRef]

50. Cao, G.L.; Sun, S.S.; Li, Z.W.; Tian, H.F.; Yang, H.X.; Li, J.Q. Clocking the anisotropic lattice dynamics of multi-walled carbon nanotubes by four-dimensional ultrafast transmission electron microscopy. Sci. Rep. 2015, 5, 8404. [CrossRef]

51. Talebi, N. Near-Field-Mediated Photon-Electron Interactions; Springer Nature AG: Gewerbestrasse, Cham, Switzerland, 2019.

52. Pomarico, E.; Madan, I.; Berruto, G.; Vanacore, G.M.; Wang, K.; Kaminer, I.; García de Abajo, F.J.; Carbone, F. $\mathrm{MeV}$ Resolution in Laser-Assisted Energy-Filtered Transmission Electron Microscopy. ACS Photonics 2018, 5, 759-764. [CrossRef]

53. Priebe, K.E.; Rathje, C.; Yalunin, S.V.; Hohage, T.; Feist, A.; Schaefer, S.; Ropers, C. Attosecond Electron Pulse Trains and Quantum State Reconstruction in Ultrafast Transmission Electron Microscopy. Nat. Photon 2017, 11, 793-797. [CrossRef]

54. Mohammed, T.H. Attomicroscopy: From femtosecond to attosecond electron microscopy. published in arXiv:1801.04350 (2018). Available online: https://arxiv.org/abs/1801.04350 (accessed on 19 May 2020).

55. Morimoto, Y.; Baum, P. Attosecond control of electron beams at dielectric and absorbing membranes. Phys. Rev. A 2018, 97, 033815. [CrossRef]

56. Morimoto, Y.; Baum, P. Diffraction and microscopy with attosecond electron pulse trains. Nat. Phys. 2018, 14, 252-256. [CrossRef]

57. Kozák, M. All-Optical Scheme for Generation of Isolated Attosecond Electron Pulses. Phys. Rev. Lett. 2019, 123, 203202. [CrossRef] [PubMed]

58. Hassan, M.T.; Baskin, J.S.; Liao, B.; Zewail, A.H. High-temporal-resolution electron microscopy for imaging ultrafast electron dynamics. Nat. Photon 2017, 11, 425-430. [CrossRef]

59. Hassan, M.T.; Luu, T.T.; Moulet, A.; Raskazovskaya, O.; Zhokhov, P.; Garg, M.; Karpowicz, N.; Zheltikov, A.M.; Pervak, V.; Krausz, F.; et al. Optical attosecond pulses and tracking the nonlinear response of bound electrons. Nature 2016, 530, 66-70. [CrossRef]

60. Madan, I.; Vanacore, G.M.; Pomarico, E.; Berruto, G.; Lamb, R.J.; McGrouther, D.; Lummen, T.T.A.; Latychevskaia, T.; García de Abajo, F.J.; Carbone, F. Holographic imaging of electromagnetic fields via electron-light quantum interference. Sci. Adv. 2019, 5, eaav8358. [CrossRef]

61. Ropers, C. Holograms from electrons scattered by light. Nature 2019, 571, 331-332. [CrossRef]

62. Plemmons, D.A.; Park, S.T.; Zewail, A.H.; Flannigan, D.J. Characterization of fast photoelectron packets in weak and strong laser fields in ultrafast electron microscopy. Ultramicroscopy 2014, 146, 97-102. [CrossRef]

63. Minogin, V.G. Identities for the electron forms 2 and their 3D representation. Ann. Phys. 2012, 327, 823-840. [CrossRef] 
64. Feist, A.; Bach, N.; Rubiano da Silva, N.; Danz, T.; Möller, M.; Priebe, K.E.; Domröse, T.; Gatzmann, J.G.; Rost, S.; Schauss, J.; et al. Ultrafast transmission electron microscopy using a laser-driven field emitter: Femtosecond resolution with a high coherence electron beam. Ultramicroscopy 2017, 176, 63-73. [CrossRef]

65. Houdellier, F.; Caruso, G.M.; Weber, S.; Kociak, M.; Arbouet, A.A. Development of a high brightness ultrafast transmission electron microscope based on a laser-driven cold field emission source. Ultramicroscopy 2018, 186, 128-138. [CrossRef]

66. Da Silva, N.R.; Möller, M.; Feist, A.; Ulrichs, H.; Ropers, C.; Schäfer, S. Nanoscale Mapping of Ultrafast Magnetization Dynamics with Femtosecond Lorentz Microscopy. Phys. Rev. X 2018, 8, 031052.

67. Möller, M.; Gaida, J.H.; Schäfer, S.; Ropers, C. Few-nm tracking of current-driven magnetic vortex orbits using ultrafast Lorentz microscopy. Commun. Phys. 2020, 3, 36. [CrossRef]

(C) 2020 by the authors. Licensee MDPI, Basel, Switzerland. This article is an open access article distributed under the terms and conditions of the Creative Commons Attribution (CC BY) license (http://creativecommons.org/licenses/by/4.0/). 